\title{
Management of insomnia in older adults
}

\author{
BØRGE SIVERTSEN and INGER HILDE NORDHUS
}

\begin{abstract}
Summary Complaints of insomnia are very common, especially in older adults. Although pharmacotherapy is the most common form of treatment, recent evidence shows cognitive-behavioural therapy to be superior in the short- and long-term management of insomnia. Lowthreshold intervention programmes may reduce both the individual and societal burden of insomnia, coexisting with or without other mental or physical disorders.
\end{abstract}

\section{Declaration of interest None.}

Insomnia is a risky business. If you are among the third of the UK population who weekly suffer from impaired sleep, you are also more likely to suffer from anxiety or depression, as well as to have impaired immune functioning. The case is particularly grave for older adults $(65+$ years), in whom the prevalence rate of insomnia doubles compared with younger adults. This age group is also by far the most loyal consumers of sedatives, including sleep medications. In terms of the economic burden of insomnia, recent analyses estimate the direct medical costs of insomnia to be around $\$ 14$ billion annually in the USA, a figure that increases to nearly $\$ 100$ billion when including indirect costs from sleep-related accidents and lost productivity.

Insomnia is also big business, especially for the pharmaceutical industry. In the past year $\$ 2$ billion were spent on sleep remedies in the USA, an estimate that will probably increase to $\$ 5$ billion within a few years. Moreover, although the use of sleeping pills doubled from 2000 to 2004 , the pharmaceutical industry still considers the sleeping pill market virgin territory, at least compared with the $\$ 11$ billion spent annually on antidepressants. Therefore, you would think the evidence backing the efficacy of sleeping pills was convincing. Not so.

In a recent meta-analysis of hypnotic drug use in older patients, representing the most prevalent age segment using prescribed drugs, the clinical benefits associated with sleep medications were found to be only small, and for some individuals actually outweighed by the adverse effects (Glass et al, 2005). Although not specifically focusing on older adults, an extensive review of the management of chronic in somnia concluded that the clinical benefits of benzodiazepine sleep aids were inflated by reporting bias (Buscemi et al, 2005).

However, at present the treatment of choice for insomnia among primary care physicians remains pharmacological intervention. This is particularly the case for older adults, for whom psychological interventions until recently generally were assumed to be less relevant and ineffective. Consequently, only a few studies have examined the effect of cognitive-behavioural interventions in older adults with insomnia. This treatment approach typically includes several components, the most common being sleep hygiene education (teaching the patient about the impact of lifestyle habits that may affect their sleep), sleep restriction (a strict schedule of bedtimes and rising times), stimulus control (instructing patients to get out of bed if they cannot fall asleep within 15-20 min), cognitive therapy (teaching the patients to identify, challenge and replace negative and erroneous thoughts related to their sleep), and relaxation techniques.

The lack of studies in this age group may in part be ascribed to the fact that older adults with insomnia are often faced with other coexisting conditions or symptoms, which according to the traditional view should be treated before any efforts be directed to the sleep problems. Although two reviews have suggested mild but promising effects of behavioural interventions on some sleep variables in this age cohort
(Montgomery \& Dennis, 2003; Irwin et $a l, 2006)$, there have until now been few efforts comparing the effects of nonpharmacological interventions with sleep medication in older adults. However, evidence from a new randomised, doubleblind placebo-controlled trial shows that interventions based on cognitive-behavioural therapy may be more effective than the newer non-benzodiazepines (zopiclone) in managing short- and long-term insomnia in older adults, as measured by polysomnography (Sivertsen et al, 2006).

Although cognitive-behavioural therapy could be regarded as the treatment of choice for late-life insomnia, an important argument against such interventions has traditionally been that the clinical effectiveness of this form of therapy in general healthcare practice is limited. Compared with pharmacological treatments for insomnia, which only involve filling a prescription and taking a tablet, cognitivebehavioural therapy usually involves long waiting lists, followed by several sessions of therapy, which are time-consuming, expensive and have ultimately been argued to be less efficacious in general practice. Thus, we consider it a fundamental challenge to identify implementation regimens that specifically address these barriers to making cognitive-behavioural interventions more available.

Interestingly, a UK study in which primary care nurses were trained to deliver cognitive-behavioural therapy to small groups of people (mean age 51 years) found this approach to be highly cost-efficient, yielding treatment effects similar to those most often are found in specialised sleep centres (Espie et al, 2001). In another study, the effectiveness of cognitive-behavioural therapy for insomnia provided by primary care counsellors was found to be well within the limits of cost-effectiveness currently thought acceptable in the UK (Morgan et al, 2003). There is also emerging evidence from studies on younger adults suggesting that other low-level treatment options for chronic insomnia may be cost-effective. For example, both telephone consultations and cognitive-behavioural group therapy were (in an uncontrolled study) found to yield improvements similar to those from individual therapy sessions (Bastien et al, 2004). In another study, self-help programmes for insomnia delivered in the context of community-based interventions demonstrated significant clinical benefits (Morin et al, 2005). Although not 
providing hard evidence, a Swedish study recently found cognitive-behavioural therapy delivered over the internet in a self-help format to be helpful in chronic insomnia, although not significantly better than a placebo treatment (Strom et al, 2004).

To date, no study has specifically explored the clinical benefits of such interventions in older adults, but we consider it likely that older people too might benefit from such treatments. As the implementation of such low-level treatment options is simple, efficient and local, we believe these interventions in the long run to be more economically sensible than chronic administration of sleep medications in general practice.

Although insomnia usually occurs together with other disorders or conditions, most treatment studies have focused on patients with little or no comorbidity. The problem of comorbidity is especially central to elderly people. It is not uncommon that older patients suffer from polypharmacy (causing unexpected drug interactions), which leaves treatment with benzodiazepines even more problematic. However, a recent review of emerging research on comorbid or secondary insomnia suggests that cognitive-behavioural therapy might be equally effective in treating people with insomnia who also have other psychiatric or medical conditions (Stepanski \& Rybarczyk, 2006). However, there is a strong need for more randomised controlled trials to establish the efficacy of cognitive-behavioural interventions in comorbid insomnia.

Future research should attempt to identify the mechanisms and specific processes involved in the development and maintenance of insomnia, as this condition is typically maintained by a range of cognitive processes regardless of any original trigger

BØRGE SIVERTSEN, PhD, INGER HILDE NORDHUS, PhD, Department of Clinical Psychology, University of Bergen, Bergen, Norway

Correspondence: Dr Børge Sivertsen, Department of Clinical Psychology, University of Bergen, Christiesgt. 12, 5015 Bergen, Norway. Email: borge.sivertsen@psykp.uib.no

(First received 22 September 2006, final revision 19 December 2006, accepted 8 January 2007)

(Harvey \& Tang, 2003). Identifying predisposing factors and refining the procedures offered in cognitive-behavioural therapy might allow us to design low-threshold intervention programmes maximising the magnitude of change associated with this form of therapy. We are now in a position where we no longer need to search for alternatives to sleep medications; the big challenge now is implementing these cognitive-behavioural interventions in general practice. The need to develop educational programmes for insomnia aimed at all healthcare professions is crucial, and might ultimately reduce the all-time record of 43 million sleeping pill prescriptions in the USA last year.

\section{REFERENCES}

Bastien, C. H., Morin, C. M., Ouellet, M. C., et al (2004) Cognitive-behavioral therapy for insomnia: comparison of individual therapy, group therapy, and telephone consultations. Journal of Consulting and Clinical Psychology, 72, 653-659.

Buscemi, N., Vandermeer, B., Friesen, C., et al (2005) Manifestations and Management of Chronic Insomnia in Adults. Agency for Healthcare Research and Quality.

Espie, C. A., Inglis, S. J., Tessier, S., et al (2001) The clinical effectiveness of cognitive behaviour therapy for chronic insomnia: implementation and evaluation of a sleep clinic in general medical practice. Behaviour Research and Therapy, 39, 45-60.
Glass, J., Lanctot, K. L., Herrmann, N., et al (2005) Sedative hypnotics in older people with insomnia: metaanalysis of risks and benefits. BMI, 331, 1169.

Harvey, A. G. \& Tang, N. K. (2003) Cognitive behaviour therapy for primary insomnia: can we rest yet? Sleep Medicine Reviews, 7, 237-262.

Irwin, M. R., Cole, J. C. \& Nicassio, P. M. (2006)

Comparative meta-analysis of behavioral interventions for insomnia and their efficacy in middle-aged adults and in older adults 55+ years of age. Health Psychology, 25, $3-14$

Montgomery, P. \& Dennis, J. (2003) Cognitive behavioural interventions for sleep problems in adults aged 60+. Cochrane Database of Systematic Reviews, issue 2. Update Software.

Morgan, K., Dixon, S., Mathers, N., et al (2003) Psychological treatment for insomnia in the management of long-term hypnotic drug use: a pragmatic randomised controlled trial. British Journal of General Practice, 53, 923-928.

Morin, C., Beaulieu-Bonneau, S., LeBlanc, M., et a (2005) Self-help treatment for insomnia: a randomized controlled trial. Sleep, 28, 1319-1327.

Sivertsen, B., Omvik, S., Pallesen, S., et al (2006) Cognitive behavioral therapy vs zopiclone for treatment of chronic primary insomnia in older adults: a randomized controlled trial. JAMA, 295, 285I-2858.

Stepanski, E. J. \& Rybarczyk, B. (2006) Emerging research on the treatment and etiology of secondary or comorbid insomnia. Sleep Medicine Reviews, 10, 7-18.

Strom, L., Pettersson, R. \& Andersson, G. (2004) Internet-based treatment for insomnia: a controlled evaluation. Journal of Consulting and Clinical Psychology, 72, $113-120$. 